\title{
Análisis del grado de satisfacción de los adultos mayores de las provincias de Azuay y Cañar
}

\author{
José Ortiz S. (D), Ulises Freire A., Marlene Álvarez S., David Tenemaza A., Ignacio Cobos T., \\ Martha Zhindón G., Eulalia Larriva V. \\ Facultad de Ciencias Médicas, Universidad de Cuenca, Cuenca, Ecuador. \\ Autor para correspondencia: jose.ortiz@ucuenca.edu.ec
}

Fecha de recepción: 13 de septiembre 2017 - Fecha de aceptación: 6 de noviembre 2017

\section{RESUMEN}

Objetivo. Analizar las percepciones sobre el ejercicio de los derechos de los adultos mayores (AM) en las provincias de Azuay y Cañar, Ecuador.

Metodología. Se realizó un estudio transversal analítico que incluyó a 538 AM que viven en las áreas de cobertura de los centros de salud de primer nivel, pertenecientes al Ministerio de Salud Pública y al Seguro Social Campesino, mediante la aplicación de un formulario previamente validado.

Resultados. La edad promedio fue de $75.20 \pm 7.22$ años (de 65 a 100 años), la mayoría fueron mujeres, casadas, con educación primaria y un 33\% de analfabetismo. El 81\% de participantes consideran como adecuado su estándar de vida. Vivir solo es un factor asociado con el ejercicio inadecuado de los derechos de una manera estadísticamente significativa (OR 2.31; IC 95\% 1.46-3.68). La edad, estado civil, ocupación, educación, auto-identificación, se encuentran también asociados con el grado de satisfacción, pero no de manera significativa.

Conclusiones. Ocho de cada diez AM consideran que el ejercicio de sus derechos es adecuado, y en el grupo que sienten insatisfacción, la soledad constituye un factor asociado de manera estadísticamente significativa. Esta situación da cuenta de la importancia de generar redes sociales de apoyo entre pares, con apoyo interinstitucional y en especial de las organizaciones comunitarias locales, para conseguir el ejercicio pleno de los derechos, sin exclusiones.

Palabras clave: Derechos del adulto mayor.

\section{ABSTRACT}

Objective. Analysis of the perceptions about the exercise of the rights of the elderly in the provinces of Azuay and Cañar.

Methodology. An analytical cross-sectional study was carried out involving 538 elderly people living in the coverage areas of the first-level health centers, belonging to the Ministry of Public Health and the Rural Social Security, through the application of a rights form, previously validated.

Results. The mean age was $75.20 \pm 7.22$ years (65 to 100 years), the majority were women, married, with primary education and 33\% illiteracy. $81 \%$ of participants consider their standard of living to be adequate. Living alone is a factor associated with the inadequate exercise of rights in a statistically significant way (OR 2.31, 95\% CI 1.46-3.68). Age, marital status, occupation, education, selfidentification, are also associated with inadequate exercise of rights, but not significantly.

Conclusions. Eight out of ten older adults consider that the exercise of their rights is adequate, and in the group that feels dissatisfaction, solitude is a statistically significant associated factor. This situation reveals the importance of generating social networks of support between peers, with inter-institutional support and especially of local community organizations, to achieve the full exercise of rights, without exclusions.

Keywords: Elder rights. 


\section{INTRODUCCIÓN}

Según la Organización Panamericana de la Salud (OPS), para el año 2020 se espera que en la región de las Américas, se duplique el número de personas mayores de 60 años con respecto a los 100 millones existentes en 2006 (Organización Panamericana de la Salud, \& Organización Mundial de la Salud, s.f.). En el Ecuador, de acuerdo con las proyecciones realizadas por el Instituto Ecuatoriano de Estadísticas y Censos (INEC), la esperanza de vida de 75 años estimada en el año 2010, subirá a 80.5 años para el 2050 (INEC, 2012). Según el último censo, de los 14’483,499 habitantes, 940,905 son mayores de 65 años que representa el $6.5 \%$ de la población nacional, con un 53\% para las mujeres y $47 \%$ para los hombres; el porcentaje de población de AM en la ciudad de Cuenca alcanzó al 6.67\%, siendo levemente mayor que la del país (6.5\%) y algo inferior que la de la provincia del Azuay que es de 7.84\% (Instituto Nacional de Estadística y Censos, 2012). Si bien esto representa un éxito de las políticas de salud pública en los últimos años, también significa un aumento de las enfermedades crónicas, las discapacidades y la necesidad de realizar investigaciones para mejorar el conocimiento y desarrollar acciones para afrontar dichas necesidades.

Durante la última década, varias instituciones nacionales e internacionales han demostrado su preocupación por la situación de las personas mayores desde la perspectiva de los derechos humanos, lo cual se ha reflejado en la adopción de un serie de políticas, planes y programas que orientan el desarrollo de actividades dirigidas al mejoramiento de la calidad de vida y salud de este grupo etario (CEPAL, UNFPA, \& ASDI, 2011).

El ejercicio parcial o incompleto de los derechos del adulto mayor ya sea por omisión o desconocimiento, constituye uno de las factores fundamentales que puede acelerar el deterioro de la calidad de vida, pues conforme avanza la edad de la población se incrementan los problemas de salud, enfermedades crónicas y la limitación funcional, con lo cual se afecta la independencia y la autonomía; en varios estudios se evidencia que la limitación funcional está asociada a factores demográficos, socioeconómicos y a patologías crónicas (Menéndez Jiménez, Guevara González, Arcia, León Díaz, Marín et al., 2014; Lara-Jaque, Pinto-Santuber, \& Espinoza-Lavoz, 2010; Soler, Mellinas, Sánchez, \& Jiménez, 2010); otras trabajos describen que la fragilidad en las personas mayores constituye un cuadro clínico que conlleva un riesgo aumentado de la limitación funcional que, asociado a comorbilidad y a discapacidad, puede presentarse de forma independiente (Fried, Tangen, Walston, Newman, Hirsch et al., 2001; Ottenbacher, Ostir, Peek, Snih, Raji et al., 2005). En este marco, la falta de información sobre los derechos de los AM, alimentación saludable, actividad física y prevención de complicaciones de las enfermedades no transmisibles, no solamente por parte de los propios AM sino de los familiares, líderes comunitarios y del personal que brinda atención de salud, constituyen barreras que impiden conseguir bienestar durante el envejecimiento, de ahí la necesidad de profundizar en el conocimiento sobre el grado de satisfacción de la población adulta mayor.

\section{MÉTODO Y MATERIALES}

Se trata de un estudio de corte transversal en el que participaron, docentes y estudiantes del ciclo Comunitario del Internado Rotativo y de la carrera de Terapia Física, correspondiente al período lectivo septiembre de 2016 a febrero de 2017.

La variables incluidas en el estudio fueron: características demográficas (edad, sexo, estado civil, con quien vive, residencia), socioeconómicas (auto-identificación, educación formal, ocupación, vivienda, servicios básicos) conocimientos y prácticas sobre derechos del adulto mayor (estándar de vida, seguro social, asistencia y protección, no discriminación, salud, trato con dignidad, protección ante rechazo o abuso mental, participación social y partición en la toma de decisiones concernientes a su bienestar) 
El estudio se realizó en las áreas de cobertura de 36 Unidades Asistenciales Docentes (UADs), del Ministerio de Salud Pública (27) y del Seguro Social Campesino (9); en las provincias de Azuay (32), y Cañar (4), donde los estudiantes de la Carrera de Medicina realizaron la rotación del ciclo comunitario.

El universo estuvo conformado por 84,218 AM de las provincias de Azuay y Cañar, según datos proyectados por el INEC (2012). El cálculo de la muestra se realizó considerando una frecuencia esperada de insatisfacción en el ejercicio de los derechos (mal trato) del 30\% (Gobierno de Chile, 2011), con un límite de confianza del 4\%, y para un nivel de confianza de 95\% se obtuvo un tamaño mínimo de la muestra de 538 AM. Los participantes fueron seleccionados en base a los siguientes criterios de inclusión: 65 años y más de edad, de ambos sexos, sin deterioro cognitivo, quienes expresaron su voluntad de participar y firmaron un consentimiento informado.

La recolección de datos fue realizada por los estudiantes del ciclo comunitario, previo un proceso de capacitación, de fuentes primarias y secundarias, mediante entrevistas estructuradas, que fueron validadas con anterioridad (alfa de Cronbach 0.73). Para garantizar la calidad de la información los docentes y tutores supervisaron el llenado de los formularios y constataron la veracidad de la información, revisando las historias clínicas de los archivos de los centros de salud. El análisis descriptivo de las variables del estudio se realizó mediante frecuencias y porcentajes, y para establecer los factores asociados, se utilizó el Odds Ratio con su intervalo de confianza al 95\% y la prueba de Chi Cuadrado, en el programa SPSS v 20.

\section{RESULTADOS}

Fueron involucrados 538 AM, la mayoría perteneciente al grupo de 65 a 79 años de edad y del sexo femenino. Al momento de la encuesta, el estado civil de casados fue para algo más de la mitad de AM, seguido de viudos, solteros, divorciados y muy pocos de unión libre. Nueve de cada diez individuos se autoidentificaron como mestizos, el resto como indígenas, blancos, negros y mulatos. Cerca de la mitad de los participantes viven con esposo o esposa, alrededor de la quinta parte viven con hijos o solos, y en menor proporción con otros familiares, hermanos y otras personas. En cuanto a la educación formal, la mayoría han cursado la primaria, la tercera parte no tiene ningún grado de instrucción, muy pocos han conseguido estudios secundarios y de tercer nivel. Ocho de cada diez AM ha manifestado que están desocupados, más un pequeño grupo que ha referido inactividad y solamente uno de cada diez ha manifestado que tienen algún trabajo remunerado (ver Tabla 1).

En la Tabla 2 se exponen los diferentes niveles de satisfacción de los AM en cuanto a sus necesidades. Podemos ver que tres de cada diez no se alimentan siempre todos los días; una tercera parte come pan, cereales, vegetales, frutas y leche; y en un porcentaje similar, no siempre le gusta lo que come.

Cerca de las tres cuartas partes de las personas mayores consideraron que siempre sus viviendas estuvieron cómodas, seguras y agradables. En cuanto a la vestimenta, casi ocho de cada diez manifestaron que la ropa que usan siempre fue cómoda, limpia, les protege y les agrada. Con respecto a la asistencia y seguridad social, solamente las dos terceras partes de los AM van al médico cuando necesitan y son jubilados; en este ámbito lo que llama la atención es que cuatro de cada diez consideran que nunca les atiende un médico.

En lo referente a la no discriminación, tres de cada diez AM consideran que no siempre los familiares y en la comunidad les tratan bien y más de la mitad dijeron que no siempre en las instituciones les brindan un buen trato.

$\mathrm{Al}$ indagar sobre los estándares de salud, si bien ocho de cada diez refirieron que los médicos les tratan con respeto, cerca de la mitad indicaron que no siempre participan en decisiones de los tratamientos, toman en cuenta su opinión en la atención o les resulta fácil obtener información para cuidar la salud; alrededor de la tercera parte no siempre acude a chequeo general, recibe información sobre su enfermedad, les proporcionan medicación necesaria, recibe orientación del médico y continúa el tratamiento. 
Tabla 1. Características demográficas y socioeconómicas de los participantes.

\begin{tabular}{|c|c|c|}
\hline Edad & $n=538$ & $\%$ \\
\hline 65 a 79 & 392 & 72.86 \\
\hline 80 a 84 & 73 & 13.57 \\
\hline 85 y más & 73 & 13.57 \\
\hline \multicolumn{3}{|l|}{ Sexo } \\
\hline Masculino & 174 & 32.34 \\
\hline Femenino & 364 & 67.66 \\
\hline \multicolumn{3}{|l|}{ Estado civil } \\
\hline Soltero & 58 & 10.78 \\
\hline Casado & 281 & 52.23 \\
\hline Viudo & 168 & 31.23 \\
\hline Divorciado & 24 & 4.46 \\
\hline Unión libre & 7 & 1.30 \\
\hline \multicolumn{3}{|l|}{ Auto-identificación } \\
\hline Indígena & 21 & 3.90 \\
\hline Negro & 1 & 0.19 \\
\hline Mestizo & 504 & 93.68 \\
\hline Mulato & 1 & 0.19 \\
\hline Blanco & 3 & 0.56 \\
\hline No sabe & 8 & 1.49 \\
\hline \multicolumn{3}{|l|}{ Con quien vive } \\
\hline Vive solo/a & 102 & 18.96 \\
\hline Con esposo/a & 243 & 45.17 \\
\hline Con hijos & 123 & 22.86 \\
\hline Con madre & 6 & 1.12 \\
\hline Con otras personas & 16 & 2.97 \\
\hline Con hermanos/as & 17 & 3.16 \\
\hline Con otros familiares & 31 & 5.76 \\
\hline \multicolumn{3}{|l|}{ Educación formal } \\
\hline Ninguna & 177 & 32.90 \\
\hline Centro de alfabetización & 33 & 6.13 \\
\hline Primaria & 310 & 57.62 \\
\hline Secundaria & 14 & 2.60 \\
\hline Tercer nivel & 2 & 0.37 \\
\hline No sabe & 2 & 0.37 \\
\hline \multicolumn{3}{|l|}{ Ocupación } \\
\hline Trabajador de los servicios y comerciante & 8 & 1.49 \\
\hline Trabajador calificado agropecuario y pesquero & 15 & 2.79 \\
\hline Oficiales operarios y artesanos & 16 & 2.97 \\
\hline Operadores de instalaciones y máquinas & 2 & 0.37 \\
\hline Trabajadores no calificados & 32 & 5.95 \\
\hline Desocupados & 432 & 80.30 \\
\hline Inactivos & 33 & 6.13 \\
\hline
\end{tabular}

Acerca del trato con dignidad, cuatro de cada diez manifiestan que no siempre les atienden pronto y con respeto; y en cuanto a la protección, casi la mitad no siempre se sienten protegidos ante el rechazo o mal trato.

Con respecto a la participación, entre la tercera parte y la mitad de AM percibieron que no siempre votan en las elecciones por su propia decisión o que se llevan bien con familiares, amigos y vecinos; además, en esta misma proporción, también manifestaron que votan en las elecciones, utilizan su dinero, 
tienen ingreso por trabajo, bono o jubilación. Entre algo menos de la mitad y la quinta parte aseguraron que no siempre se sienten contentos, han conseguido lo que se han propuesto, pertenecen a alguna organización, cuentan con espacios de distracción comunitaria, participan en decisiones y en obras

Tabla 2. Percepciones de los adultos mayores sobre el ejercicio de sus derechos.

\begin{tabular}{|c|c|c|c|c|c|c|c|c|c|c|}
\hline \multirow{2}{*}{$\begin{array}{l}\text { Alimentación } \\
\text { Se alimenta todos los días }\end{array}$} & \multicolumn{2}{|c|}{$\begin{array}{c}\text { Siempre } \\
\text { N (\%) }\end{array}$} & \multicolumn{2}{|c|}{$\begin{array}{c}\text { Casi siempre } \\
\mathrm{N}(\%) \\
\end{array}$} & \multicolumn{2}{|c|}{$\begin{array}{c}\text { Algunas veces } \\
\text { N (\%) } \\
\end{array}$} & \multicolumn{2}{|c|}{$\begin{array}{l}\text { Casi nunca } \\
\quad \mathrm{N}(\%) \\
\end{array}$} & \multicolumn{2}{|c|}{$\begin{array}{l}\text { Nunca } \\
\text { N (\%) }\end{array}$} \\
\hline & 368 & $(68.40)$ & 110 & $(20.45)$ & 52 & $(9.67)$ & 8 & $(1.49)$ & 0 & $(0.00)$ \\
\hline Come: pan, cereales, vegetales, frutas, leche & 184 & $(34.20)$ & 172 & (31.97) & 142 & $(26.39)$ & 35 & $(6.51)$ & 5 & $(0.93)$ \\
\hline Le gusta lo que come & 356 & $(66.17)$ & 108 & $(20.07)$ & 57 & $(10.59)$ & 15 & $(2.79)$ & 2 & $(0.37)$ \\
\hline \multicolumn{11}{|l|}{ Vivienda } \\
\hline Es cómoda & 392 & (72.86) & 92 & $(17.10)$ & 43 & $(7.99)$ & 6 & $(1.12)$ & 5 & $(0.93)$ \\
\hline Es segura & 371 & (68.96) & 107 & (19.89) & 40 & $(7.43)$ & 17 & (3.16) & 3 & $(0.56)$ \\
\hline Le agrada & 400 & $(74.35)$ & 90 & $(16.73)$ & 40 & $(7.43)$ & 4 & $(0.74)$ & 4 & $(0.74)$ \\
\hline \multicolumn{11}{|l|}{ Vestimenta } \\
\hline Es cómoda & 428 & (79.55) & 74 & $(13.75)$ & 34 & $(6.32)$ & 2 & $(0.37)$ & 0 & $(0.00)$ \\
\hline Está limpia & 416 & (77.32) & 90 & (16.73) & 25 & $(4.65)$ & 4 & $(0.74)$ & 3 & $(0.56)$ \\
\hline Le protege del frío, calor y viento & 425 & (79.00) & 85 & $(15.80)$ & 22 & $(4.09)$ & 3 & $(0.56)$ & 3 & $(0.56)$ \\
\hline Le agrada & 429 & $(79.74)$ & 70 & $(13.01)$ & 36 & $(6.69)$ & 2 & $(0.37)$ & 1 & $(0.19)$ \\
\hline \multicolumn{11}{|l|}{ Asistencia y seguridad social } \\
\hline Va donde el médico cuando necesita & 347 & $(64.50)$ & 106 & $(19.70)$ & 63 & $(11.71)$ & 13 & $(2.42)$ & 9 & $(1.67)$ \\
\hline Es jubilado & 353 & $(65.61)$ & 112 & $(20.82)$ & 34 & $(6.32)$ & 12 & $(2.23)$ & 27 & $(5.02)$ \\
\hline Le atiende el médico & 282 & $(52.42)$ & 31 & $(5.76)$ & 12 & $(2.23)$ & 4 & $(0.74)$ & 209 & $(38.85)$ \\
\hline \multicolumn{11}{|l|}{ No discriminación } \\
\hline Los familiares le tratan bien & 374 & $(69.52)$ & 91 & (16.91) & 57 & $(10.59)$ & 9 & $(1.67)$ & & $(1.30)$ \\
\hline En la comunidad le tratan bien & 387 & (71.93) & 84 & (15.61) & 55 & $(10.22)$ & 8 & $(1.49)$ & 4 & $(0.74)$ \\
\hline En las instituciones le tratan bien & 325 & $(43.45)$ & 325 & $(43.45)$ & 60 & $(8.02)$ & 19 & $(2.54)$ & 19 & $(2.54)$ \\
\hline \multicolumn{11}{|l|}{ Estándares de salud } \\
\hline Acude a chequ & 333 & $(61.90)$ & 107 & $(19.89)$ & 62 & $(11.52)$ & 26 & $(4.83)$ & 10 & $(1.86)$ \\
\hline Continúa el tratamiento & 389 & $(72.30)$ & 84 & (15.61) & 47 & $(8.74)$ & 7 & $(1.30)$ & 11 & $(2.04)$ \\
\hline Participa en decisiones de tratamientos & 285 & $(52.97)$ & 123 & $(22.86)$ & 82 & $(15.24)$ & 29 & (5.39) & 19 & (3.53) \\
\hline Recibe orientación del médico & 383 & (71.19) & 98 & (18.22) & 42 & $(7.81)$ & 9 & $(1.67)$ & 6 & $(1.12)$ \\
\hline El médico le atiende con respeto & 431 & $(80.11)$ & 80 & (14.87) & 20 & $(3.72)$ & 4 & $(0.74)$ & 3 & $(0.56)$ \\
\hline Recibe información sobre su enfermedad & 361 & $(67.10)$ & 107 & (19.89) & 44 & $(8.18)$ & 18 & $(3.35)$ & 8 & $(1.49)$ \\
\hline Le proporcionan medicación necesaria & 368 & $(68.40)$ & 106 & (19.70) & 47 & $(8.74)$ & 7 & $(1.30)$ & 10 & $(1.86)$ \\
\hline $\begin{array}{l}\text { Fácil obtener información para cuidar la } \\
\text { salud }\end{array}$ & 294 & (54.6 & 121 & (22. & 74 & 13 & 31 & (5.76) & 18 & (3.35) \\
\hline Toman en cuenta su opinión en la atención & 287 & $(53.35)$ & 121 & $(22.49)$ & 87 & $(16$. & 25 & $(4.65)$ & 18 & $(3.35)$ \\
\hline \multicolumn{11}{|l|}{ Trato con dignidad } \\
\hline Le atienden $\mathrm{p}$ & 367 & $(68.22)$ & 113 & $(21.00)$ & 44 & $(8.18)$ & 7 & $(1.30)$ & 7 & $(1.30)$ \\
\hline \multicolumn{11}{|l|}{ Protección } \\
\hline Se siente protegido ante recha & 287 & $(53.35)$ & 127 & $(23.61)$ & 80 & $(14.87)$ & 19 & $(3.53)$ & 25 & $(4.65)$ \\
\hline \multicolumn{11}{|l|}{ Participación comunitaria } \\
\hline Participa en obras comunitarias & 107 & (19.89) & 57 & (10.59) & 96 & $(17.84)$ & 81 & $(15.06)$ & 197 & $(36.62)$ \\
\hline Participa en decisiones comunitarias & 109 & $(20.26)$ & 61 & (11.34) & 115 & $(21.38)$ & 84 & $(15.61)$ & 169 & (31.41) \\
\hline Capacita a otras personas & 78 & $(14.50)$ & 51 & $(9.48)$ & 87 & $(16.17)$ & 80 & $(14.87)$ & 242 & $(44.98)$ \\
\hline Pertenece a alguna organización & 142 & (26.39) & 33 & (6.13) & 25 & $(4.65)$ & 27 & $(5.02)$ & 311 & $(57.81)$ \\
\hline Tiene ingreso por trabajo, bono o jubilación & 291 & (54.09) & 29 & (5.39) & 43 & $(7.99)$ & 22 & $(4.09)$ & 153 & $(28.44)$ \\
\hline Utiliza su dinero & 309 & $(57.43)$ & 61 & $(11.34)$ & 98 & $(18.22)$ & 32 & $(5.95)$ & 38 & $(7.06)$ \\
\hline Apoya a otras personas con su dinero & 86 & (15.99) & 55 & $(10.22)$ & 76 & $(14.13)$ & 63 & $(11.71)$ & 258 & $(47.96)$ \\
\hline Vota en las elecciones & 321 & (59.67) & 43 & (7.99) & 27 & $(5.02)$ & 20 & $(3.72)$ & 127 & $(23.61)$ \\
\hline Vota por su propia decisión & 374 & $(69.52)$ & 26 & $(4.83)$ & 17 & $(3.16)$ & 9 & $(1.67)$ & 112 & $(20.82)$ \\
\hline Ha orientado o aconsejado a los gobernantes & 81 & $(15.06)$ & 27 & $(5.02)$ & 55 & $(10.22)$ & 60 & $(11.15)$ & 315 & $(58.55)$ \\
\hline Participa en actividades sociales, culturales & 84 & $(15.61)$ & 42 & $(7.81)$ & 99 & $(18.40)$ & 79 & (14.68) & 234 & $(43.49)$ \\
\hline Espacios de distracción comunitaria & 122 & $(22.68)$ & 49 & $(9.11)$ & 93 & $(17.29)$ & 63 & $(11.71)$ & 211 & $(39.22)$ \\
\hline Ha encontrado programas para su desarrollo & 42 & $(7.81)$ & 31 & $(5.76)$ & 95 & $(17.66)$ & 103 & (19.14) & 267 & (49.63) \\
\hline Maneja computadora, celular, cajero & 22 & $(4.09)$ & 12 & $(2.23)$ & 40 & $(7.43)$ & 37 & $(6.88)$ & 427 & $(79.37)$ \\
\hline Se siente contento/a feliz, alegre & 247 & $(45.91)$ & 138 & $(25.65)$ & 117 & $(21.75)$ & 29 & (5.39) & 7 & $(1.30)$ \\
\hline Ha conseguido lo que se ha propuesto & 208 & (38.66) & 141 & (26.21) & 130 & $(24.16)$ & 37 & (6.88) & 22 & $(4.09)$ \\
\hline $\begin{array}{l}\text { Se lleva bien con familiares, amigos, } \\
\text { vecinos }\end{array}$ & 374 & (69.52) & 119 & (22.12) & 31 & $(5.76)$ & 9 & (1.67) & 5 & $(0.93)$ \\
\hline
\end{tabular}


comunitarias. Menos de la quinta parte, ha señalado que no siempre apoyan a otras personas con su dinero, participan en actividades sociales y culturales, han orientado o aconsejado a los gobernantes, capacitan a otras personas, han encontrado programas para su desarrollo y manejan computadora, celular y cajero automático.

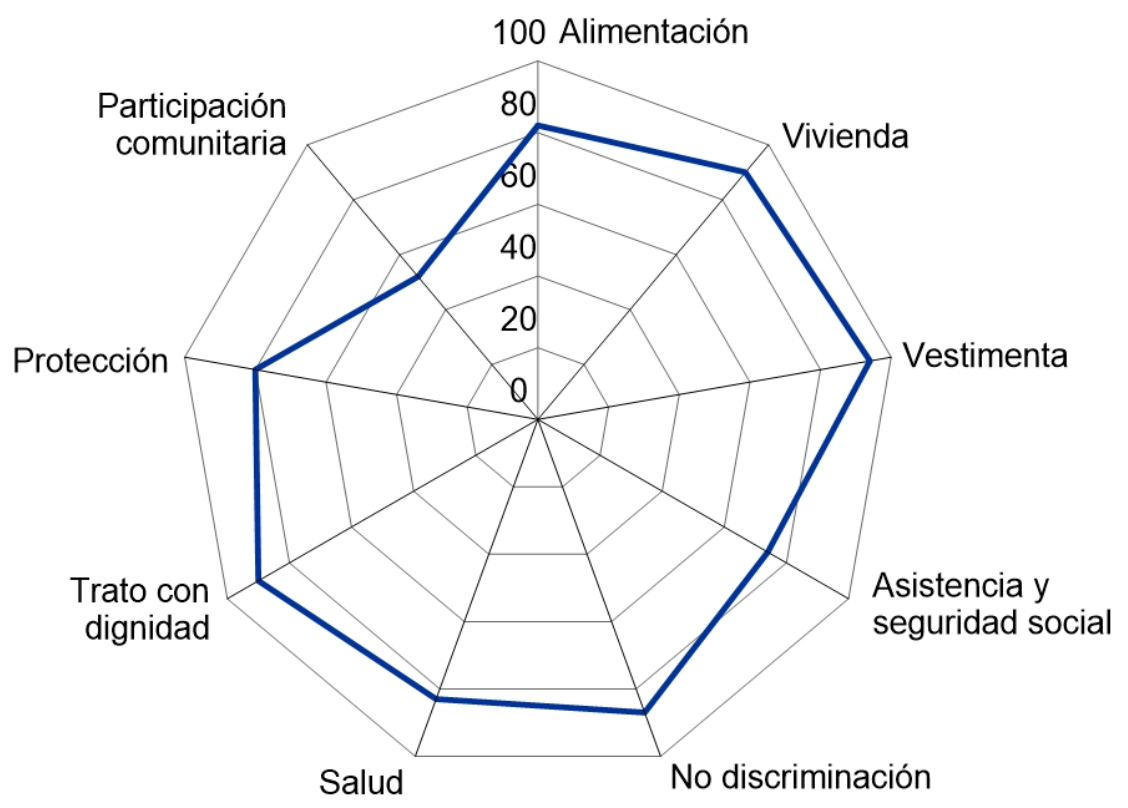

Figura 1. Percepciones de los adultos mayores sobre sus estándares de vida.

En la Figura 1 podemos apreciar, en síntesis, que la percepción de los AM sobre estándares de vida fue: para alimentación $83 \%$, vivienda $90 \%$, vestimenta $93 \%$, asistencia y seguridad social $76 \%$, no discriminación $87 \%$, salud $85 \%$, trato con dignidad $88 \%$, protección $79 \%$, participación comunitaria $50 \%$, con un promedio del $81 \%$ para el ejercicio de todos los estándares en general.

En la Tabla 3 se exponen los factores asociados con el ejercicio inadecuado de los derechos; en este sentido, encontramos que la edad, estado civil, ocupación, educación, se encuentran relacionados, pero no en forma significativa. En tanto que el hecho de vivir solo o sola, fue identificado como un factor asociado de manera estadísticamente significativa (OR 2.31; IC 95\% 1.46-3.68).

Tabla 3. Factores asociados al ejercicio inadecuado de los derechos.

\begin{tabular}{|c|c|c|c|c|c|c|c|c|}
\hline \multirow{3}{*}{$\begin{array}{l}\text { Variables asociadas } \\
\text { Edad }\end{array}$} & \multicolumn{8}{|c|}{ Ejercicio de todos los derechos } \\
\hline & \multicolumn{2}{|c|}{ Inadecuado } & \multicolumn{2}{|c|}{ Adecuado } & \multirow{2}{*}{ OR } & \multicolumn{2}{|c|}{ IC $95 \%$} & \multirow{2}{*}{ Valor P } \\
\hline & No & $\%$ & No & $\%$ & & Inf & Sup & \\
\hline 65 a 79 & 92 & 23.47 & 300 & 76.53 & 0.97 & 0.62 & 1.52 & 0.90 \\
\hline 80 a 84 & 15 & 20.55 & 58 & 79.45 & 0.82 & 0.44 & 1.49 & 0.51 \\
\hline 85 y más & 20 & 27.40 & 53 & 72.60 & 1.26 & 0.72 & 2.21 & 0.41 \\
\hline \multicolumn{9}{|l|}{ Sexo } \\
\hline Masculino & 34 & 19.54 & 140 & 80.46 & 0.71 & 0.45 & 1.10 & 0.12 \\
\hline Femenino & 93 & 25.55 & 271 & 74.45 & 1.41 & 0.91 & 2.20 & 0.12 \\
\hline \multicolumn{9}{|l|}{ Estado Civil } \\
\hline Soltero & 17 & 29.31 & 41 & 70.69 & 1.39 & 0.76 & 2.55 & 0.28 \\
\hline Casado & 62 & 22.06 & 219 & 77.94 & 0.84 & 0.56 & 1.25 & 0.38 \\
\hline
\end{tabular}




\begin{tabular}{lcccccccc} 
Viudo & 38 & 22.62 & 130 & 77.38 & 0.92 & 0.60 & 1.42 & 0.72 \\
Divorciado & 8 & 33.33 & 16 & 66.67 & 1.66 & 0.69 & 3.97 & 0.25 \\
Unión libre & 2 & 28.57 & 5 & 71.43 & 1.30 & 0.25 & 6.78 & 0.76 \\
\hline
\end{tabular}

Tabla 3. (Continuación)

\begin{tabular}{|c|c|c|c|c|c|c|c|c|}
\hline \multirow{3}{*}{$\begin{array}{l}\text { Variables asociadas } \\
\text { Con quien vive }\end{array}$} & \multicolumn{8}{|c|}{ Ejercicio de todos los derechos } \\
\hline & \multicolumn{2}{|c|}{ Inadecuado } & \multicolumn{2}{|c|}{ Adecuado } & \multirow{2}{*}{ OR } & \multicolumn{2}{|c|}{ IC 95\% } & \multirow{2}{*}{ Valor P } \\
\hline & No & $\%$ & No & $\%$ & & Inf & Sup & \\
\hline Vive solo/a & 38 & 37.25 & 64 & 62.75 & 2.31 & 1.46 & 3.68 & 0.0003 \\
\hline Con esposo/a & 52 & 21.40 & 191 & 78.60 & 0.80 & 0.53 & 1.20 & 0.27 \\
\hline Con hijos & 28 & 22.76 & 95 & 77.24 & 0.94 & 0.58 & 1.52 & 0.80 \\
\hline Con madre & 0 & 0.00 & 6 & 100.0 & - & - & - & 0.17 \\
\hline Con otras personas & 2 & 12.50 & 14 & 87.50 & 0.45 & 0.10 & 2.02 & 0.29 \\
\hline Con hermanos/as & 4 & 23.53 & 13 & 76.47 & 1.00 & 0.32 & 3.11 & 0.99 \\
\hline Con otros familiares & 3 & 9.68 & 28 & 90.32 & 0.33 & 0.10 & 1.11 & 0.06 \\
\hline \multicolumn{9}{|l|}{ Ocupación } \\
\hline Trabajador de los servicios y comerciante & 0 & 0.00 & 8 & 100.0 & - & - & - & 0.11 \\
\hline Trabajador calificado agropecuario y pesquero & 4 & 26.67 & 11 & 73.33 & 1.18 & 0.37 & 3.78 & 0.78 \\
\hline Oficiales operarios y artesanos & 4 & 25.00 & 12 & 75.00 & 1.08 & 0.34 & 3.41 & 0.89 \\
\hline Operadores de instalaciones y máquinas & 1 & 50.00 & 1 & 50.00 & 3.25 & 0.20 & 52.40 & 0.38 \\
\hline Trabajadores no calificados & 4 & 12.50 & 28 & 87.50 & 0.44 & 0.15 & 1.29 & 0.13 \\
\hline Desocupados & 105 & 24.31 & 327 & 75.69 & 1.23 & 0.73 & 2.06 & 0.44 \\
\hline Inactivos & 9 & 27.27 & 24 & 72.73 & 1.23 & 0.56 & 2.72 & 0.61 \\
\hline \multicolumn{9}{|l|}{ Nivel de instrucción } \\
\hline Ninguno & 49 & 27.68 & 128 & 72.32 & 1.39 & 0.92 & 2.10 & 0.12 \\
\hline Centro de alfabetización & 11 & 33.33 & 22 & 66.67 & 1.68 & 0.79 & 3.56 & 0.17 \\
\hline Primaria & 65 & 20.97 & 245 & 79.03 & 0.71 & 0.48 & 1.06 & 0.09 \\
\hline Secundaria & 1 & 7.14 & 13 & 92.86 & 0.24 & 0.03 & 1.88 & 0.14 \\
\hline Tercer nivel & 0 & 0.00 & 2 & 100.0 & - & - & - & 0.43 \\
\hline No sabe & 1 & 50.00 & 1 & 50.00 & 3.25 & 0.20 & 52.40 & 0.38 \\
\hline
\end{tabular}

Fuente: formularios de recolección de datos

\section{DISCUSIÓN}

Los derechos humanos son aquellas condiciones instrumentales o medios que le permiten a las personas su realización, sin distinción alguna de raza, color, sexo, idioma, religión, opinión política o de cualquier otra índole, origen nacional o social, posición económica, nacimiento o cualquier otra condición (UNESCO, 2008). En nuestro estudio el $20 \%$ de AM considera que no siempre pueden ejercer sus derechos, lo cual se ha evidenciado mediante el grado de satisfacción. Se pudo evidenciar que los derechos se ejercen de manera diferente, lo cual está influenciado por la edad, sexo, estado civil, vivir con otras personas, ocupación y el nivel educativo, aunque dichas diferencias no fueron estadísticamente significativas.

Si bien los derechos, identificados en la literatura (Naciones Unidas, Oficina del Alto Comisionado s.f.), se consideran como indivisibles, interdependientes e interrelacionados, en nuestros resultados hemos identificado que no todos se ejercen completamente; así, podemos ver que por ejemplo con la vestimenta, nueve de cada diez participantes se encuentran satisfechos, pero la participación comunitaria únicamente la ejercen siempre un 50\% de AM.

Existen una serie de compromisos internacionales, tales como: el Plan de Acción sobre el envejecimiento, teniendo como referente la Declaración Universal de los Derechos Humanos; la Declaración de Hong Kong de la Asociación Médica Mundial sobre el maltrato de los ancianos que se llevó acabo en la 41 Asamblea Médica Mundial (septiembre, 1989), revisada en la 126a Sesión del Consejo Jerusalén (mayo, 1990) y en la 170a Sesión del Consejo de Divonne-les Bains en Francia 
(mayo, 2005); la Estrategia regional de implementación para América Latina y el Caribe del Plan de Acción Internacional de Madrid sobre el Envejecimiento establecida por los Estados Miembros de la CEPAL, la Declaración de Brasilia. Así mismo en el marco legal nacional, que consta en la Constitución Política del Ecuador, Plan Nacional para el Buen Vivir 2013-2017, Ley del Anciano, Art. 17 de la Ley de Seguridad Social, se establece que el Estado garantizará a las personas adultas mayores los derechos a la atención de salud gratuita, trabajo remunerado según sus capacidades, la jubilación universal, rebajas en algunos servicios públicos y privados, exenciones en el régimen tributario, exoneración del pago por costos notariales y registrales, acceso a una vivienda, entre otros, todos orientados a asegurar una vida digna (Asamblea Constituyente, 2008; H. Congreso Nacional, 2016; Secretaría Nacional de Planificación y Desarrollo del Ecuador, 2013; Congreso Nacional, 2015); no obstante, en nuestro estudio los AM perciben que sus derechos no han sido atendidos por las instituciones del Estado, tal como se establece en el marco legal y en la serie de compromisos firmados.

En varias publicaciones se demuestra que la soledad, entendida como la sensación de no tener el afecto necesario deseado, un sentimiento doloroso, una situación no buscada por las personas mayores, produce sufrimiento, desolación, insatisfacción, angustia; además constituye un importante factor para padecer o agravar determinadas enfermedades y sufrir aislamiento social, estando muy vinculada a los estilos de vida (Bermejo, 2016; Egea Olivas, 2014; Cardona Jiménez, Villamil Gallego, Henao Villa, \& Quintero Echeverri, 2013). En nuestro estudio encontramos asociación entre la soledad con la insatisfacción; es decir, con la carencia en el ejercicio de los derechos de los AM.

\section{CONCLUSIONES}

No todos los AM tienen una percepción satisfactoria sobre sus estándares de vida, teniendo en cuenta que la totalidad de la población debería ejercer sus derechos. Entre los estándares con los que tienen un nivel más alto de satisfacción se encuentran: la vestimenta, vivienda, trato con dignidad, no discriminación, salud, protección, asistencia y seguridad social; la participación comunitaria únicamente ejerce la mitad de la población adulta mayor.

La edad, estado civil, ocupación, educación, constituyen factores relacionados con el grado de satisfacción de los AM, pero no en forma significativa. La soledad fue el único factor asociado, de manera estadísticamente significativa, con la insatisfacción de los AM.

Por lo antes expuesto, se desprende la necesidad de la conformación de redes sociales de apoyo entre pares, en donde los promotores de la salud podrían ser los propios AM que se encuentren en mejores condiciones físicas, mentales y socioculturales, contando con el apoyo de las organizaciones comunitarias y las instituciones vinculadas al sector salud.

Los resultados del presente estudio se deberían utilizar con cautela al pretender generalizar a toda la población de AM en razón de que la muestra seleccionada estuvo circunscrita al área de cobertura de los servicios de salud de primer nivel donde los estudiantes de la carrera de medicina realizan su rotación del Ciclo Comunitario, quedando fuera la población que no tiene contacto con los servicios de salud.

Se detecta la necesidad de mejorar los sistemas de registro y la calidad de la información para investigaciones futuras teniendo como base la información recolectada electrónicamente de manera permanente. Por otro lado, es necesaria la realización de nuevos estudios que tengan en cuenta las diversidades étnicas, culturales y de género, así como del impacto organizativo. Esto servirá para tener datos que sustenten la toma de decisiones de médicos que atienden a AM, de las autoridades sanitarias y de las entidades que financian investigaciones sanitarias.

\section{AGRADECIMIENTOS}

Expresamos un agradecimiento a todos los estudiantes del internado de las carreras de Medicina y de Terapia Física que contribuyeron en la recolección de datos y en la consolidación de las organizaciones 
comunitarias que viabilizaron la investigación. Del mismo modo nuestro reconocimiento a los profesionales de los centros de salud, tanto del Ministerio de Salud Pública como del Seguro Social Campesino, por su apoyo en todas las actividades que facilitaron la concurrencia de los participantes; a los AM líderes de las comunidades por su colaboración en todo el proceso del estudio. De manera especial expresamos nuestra gratitud a las autoridades de la Facultad de Ciencias Médicas de la Universidad de Cuenca por habernos facilitado el tiempo y los recursos materiales necesarios, de manera ágil y oportuna.

\section{BIBLIOGRAFÍA}

Asamblea Constituyente. (2008). Constitución de la República del Ecuador. Recuperado a partir de http://dstats.net/download/http://bivicce.corteconstitucional.gob.ec/site/image/common/libros/co nstituciones/Constitucion_2008_reformas.pdf

Bermejo, J. C. (2016). La soledad en los mayores. ARS MEDICA Revista de Ciencias Médicas, 32(2), 126-144.

Cardona Jiménez, J. L., Villamil Gallego, M. M., Henao Villa, E., Quintero Echeverri, Á. (2013). El sentimiento de soledad en adultos. Medicina UPB, 32(1), 9-19.

CEPAL, UNFPA, \& ASDI. (2011). Los derechos de las personas mayores. Materiales de estudio y divulgación. Módulo 3: Las normas y políticas regionales y nacionales sobre las personas mayores. Centro Latinoamericano y Caribeño de Demografía (CELADE) - División de Población de la Comisión Económica para América Latina y el Caribe (CEPAL). Recuperado a partir de www.cepal.org/celade/noticias/documentosdetrabajo/5/43685/Modulo__3.pdf

Congreso Nacional. (2015). Ley de Seguridad Social. Recuperado a partir de http://repositorio.unemi.edu.ec/handle/123456789/2794

Egea Olivas, J. (2014). La soledad en el proceso de envejecimiento. Recuperado a partir de http://digibug.ugr.es/handle/10481/36497

Fried, L. P., Tangen, C. M., Walston, J., Newman, A. B., Hirsch, C., Gottdiener, J., ... others. (2001). Frailty in older adults evidence for a phenotype. The Journals of Gerontology Series A: Biological Sciences and Medical Sciences, 56(3), M146-M157.

Gobierno de Chile. (2011). Ser mayor. Participación y autonomía. Servicio Nacional del Adulto Mayor. Recuperado a partir de www.senama.cl/filesapp/SERMAYOR.pdf

H. Congreso Nacional. (2016). Ley del anciamo. La Comisión de Legislación y Codificación. Recuperado a partir de www.sri.gob.ec/BibliotecaPortlet/descargar/...f98a.../ 160429+Ley+del+Anciano.pdf

INEC. (2012). ¿Cómo crecerá la población en Ecuador? Recuperado a partir de http://www.inec.gob.ec/proyecciones_poblacionales/presentacion.pdf

Instituto Nacional de Estadística y Censos. (2012). Base de Datos - Censo de Población y Vivienda. Recuperado a partir de http://www.ecuadorencifras.gob.ec/base-de-datos-censo-de-poblacion-yvivienda/

Lara-Jaque, R., Pinto-Santuber, C., Espinoza-Lavoz, E. (2010). Actividades básicas de la vida diaria en personas mayores y factores asociados. Rev Chil Salud Pública, 14(2-3), 291-310.

Menéndez Jiménez, J., Guevara González, A., Arcia, N., León Díaz, E. M., Marín, C., \& Alfonso, J. C. (2014). Enfermedades crónicas y limitación funcional en adultos mayores. Estudio comparativo en siete ciudades de América Latina y el Caribe. Recuperado a partir de http://www.repositoriocdpd.net:8080/handle/123456789/371

Naciones Unidas. Oficina del Alto Comisionado. (s. f.). ¿Qué son los derechos humanos? Recuperado a partir de http://www.ohchr.org/SP/Issues/Pages/WhatareHumanRights.aspx

Organización Panamericana de la Salud y Organización Mundial de la Salud. (s.f.). El creciente número de adultos mayores crea inquietudes particulares en materia de salud y derechos 
humanos [Dinámica]. Recuperado a partir de http://www.paho.org/hq/index.php?option=com_content\&view=article\&id=7867\%3A2012seniors-in-growing-numbers-present-special-health-human-rightsconcerns\&catid=1443\%3Aweb-bulletins\&Itemid=135\&lang=es

Ottenbacher, K. J., Ostir, G. V., Peek, M. K., Snih, S. A., Raji, M. A., Markides, K. S. (2005). Frailty in older Mexican Americans. Journal of the American Geriatrics Society, 53(9), 1524-1531. https://doi.org/10.1111/j.1532-5415.2005.53511.x

Secretaría Nacional de Planificación y Desarrollo del Ecuador. (2013). Plan Nacional del Buen Vivir. Secretaría Nacional de Planificación y Desarrollo del Ecuador. Recuperado a partir de http://www.buenvivir.gob.ec/documents/10157/13699/PPT+GABINETE+AMPLIADO.pdf

Soler, P. A., Mellinas, G. P., Sánchez, E. M., Jiménez, E. L. (2010). Evaluación de la comorbilidad en la población anciana: utilidad y validez de los instrumentos de medida. Revista Española de Geriatría y Gerontología, 45(4), 219-228.

UNESCO. (2008). Declaración Universal de Derechos Humanos. Recuperado a partir de http://unesdoc.unesco.org/images/0017/001790/179018m.pdf 